\title{
Cerebellum and brainstem vasculopathy in systemic lupus erythematosus: two clinico-pathological cases
}

\author{
R W Smith, D W Ellison, E A Jenkins, P J Gallagher, M I D Cawley
}

\begin{abstract}
The case histories are reported of two patients with systemic lupus erythematosus (SLE) who developed fatal neurological involvement, each presenting with cerebellar and brainstem signs. Neuro-pathological changes were confined to the cerebellum and brainstem, with clear evidence of a small vessel vasculopathy. The predominant histological abnormalities were multiple perivascular haemorrhages in one case and classical vasculitic changes in the other.
\end{abstract}

(Ann Rheum Dis 1994; 53: 327-330)

Neuropsychiatric disease (NP) may affect up to $75 \%$ of patients with systemic lupus erythematosus (SLE), resulting in a wide range of clinical manifestations. ${ }^{1}$ Seizures and psychiatric changes are common in NP-SLE, however, focal signs and rare instances of fulminant disease are also well recognised. Various mechanisms have been proposed, but the aetiopathogenesis of NP-SLE remains unclear. We report two patients with SLE who developed fatal neurological involvement of cerebellum and brainstem, and in whom post mortem study showed clear evidence of localised small vessel vasculopathies.

\section{Case Reports}

CASE 1

A 41 year old woman presented in 1981 with a symmetrical polyarthritis of the hands. A diagnosis of SLE was suggested by a positive ANF (IgG 1:1,280 and IgM 1:320) and raised

Results of investigations

\begin{tabular}{lll}
\hline & Case 1 & Case 2 \\
\hline Haemoglobin & $104 \mathrm{~g} / \mathrm{L}$ & $140 \mathrm{~g} / \mathrm{L}$ \\
WBC & $6 \cdot 1$ & $7 \cdot 4$ \\
Platelets & 510 & 186 \\
ESR & $120 \mathrm{~mm} / \mathrm{hour}$ & $78 \mathrm{~mm} / \mathrm{hour}$ \\
CRP & $241 \mathrm{mg} / \mathrm{L}$ & $104 \mathrm{mg} / \mathrm{L}$ \\
Blood cultures & Negative & Negative \\
Echocardiogram & Normal & Normal \\
ANF & IgG $1: 1,280$ & $1: 320$ \\
& IgM $1: 80$ & Raised $(1: 40$ by Crithidia method) \\
Anti DNA binding & 424 iu/L & - \\
Rheumatoid factor & 400 iu/L & N/A \\
aCL antibody & $20 \mathrm{GPL}$ & N/A \\
Anti-Ro antibody & Positive & N/A \\
Immune complexes & - & N/A \\
C Cnd $_{3}$ and & Normal & N/A \\
Cryoglobulins & + & N/A \\
ANCA & Negative & \\
\hline
\end{tabular}

N/A: Not available
DNA binding of $21 \%$ (normal $<16 \%$ ). Clinical remission and a fall in ESR from 40 to $10 \mathrm{~mm} /$ hour coincided with the introduction of hydroxychloroquine, which was continued until 1983. The ANF remained positive, and anti-Ro (SS-A) and anti-cardiolipin (aCL) antibodies (20 GPL) were detected. In 1987, following recurrent episodes of synovitis, azathioprine was started (150 mg/day).

In 1990, aged 50, she presented with malaise, sweats, nausea, vomiting and a history of recent weight loss. She had an intermittent fever of up to $39^{\circ} \mathrm{C}$, prominent livedo reticularis, splinter haemorrhages, a quiet ejection systolic murmur and synovitis of MCPJs and PIPJs. She was normotensive. Exhaustive investigation for infection was negative. Other results are shown in the table. Empirical antibiotic therapy was ineffective and azathioprine, a rare cause of drug induced fever, was later withdrawn, but again with no response.

Two weeks later she developed acute headache, vertical and horizontal nystagmus, dysarthria and ataxia. This was rapidly followed by peripheral nerve signs, generalised hyper-reflexia and extensor plantar responses. Emergency brain CT scan and MRI were both normal. The CSF protein level was elevated at $883 \mathrm{mg} / \mathrm{L}$, but with no evidence of local immunglobulin synthesis or immune complexes. An electroencephalogram was within normal limits. Nerve conduction and electromyography studies demonstrated a mononeuritis multiplex. Visual, brainstem auditory, and somato-sensory evoked potentials were all abnormal.

In the absence of an alternative diagnosis, the neurological features were attributed to SLE and/or antiphospholipid syndrome (APS). Although a thromboembolic aetiology could not be excluded, this was thought unlikely. Intravenous anticoagulation was therefore considered inappropriate, but she was maintained on subcutaneous heparin. Treatment with alternating daily intravenous methylprednisolone (1 gm) and cyclophosphamide (500 mg) was started, but after 11 days a dramatic and rapid deterioration occurrred. She developed tetraplegia, bulbar palsy, loss of sphincter control and required temporary ventilation. Enteral prednisolone was given (60 mg/day) together with intravenous immunoglobulin and alternate day plasmapheresis was performed. Neurological signs remained unchanged, but she became increasingly depressed and withdrawn. An obstructive 
uropathy developed and renal failure supervened despite nephrostomy tube drainage. Death resulted from bronchopneumonia.

\section{PATHOLOGY}

Necropsy findings included a bilateral basal pneumonia, renal papillary necrosis, a left iliac vein thrombosis and mild left ventricular hypertrophy. There was no sign of a LibmanSacks endocarditis, or of lupus nephritis.

The external appearance of the fresh brain (1400 gm), including the cerebral vasculature, was normal. Coronal sections of fixed brain showed haemorrhages measuring up to $7 \mathrm{~mm}$ in diameter in the midbrain, pons and right superior cerebellar peduncle.

Histological abnormalities were confined to the cerebellum and brainstem. Haemorrhages around small blood vessels were evident in the cerebellum, midbrain and pons (fig 1). Many of the vessels were disrupted and some contained fibrin thrombi, but there was no acute vasculitis. These lesions covered several separate vascular territories. Foci of infarction of several weeks duration were seen in the pons and medulla. No abnormality was found in the cerebral hemispheres.

\section{CASE 2}

A 52 year old man who was an insulin dependent diabetic presented in 1989 with episcleritis, recurrent arthralgias, intermittent atrial fibrillation and pericarditis. Subsequently he developed recurrent febrile episodes and weight loss. Results of investigation in another hospital supported a diagnosis of SLE (table). $\mathrm{He}$ was treated with prednisolone, (initially $60 \mathrm{mg} /$ day, subsequently reduced to $40 \mathrm{mg} /$ day), azathioprine ( $200 \mathrm{mg} /$ day), with the addition, in May 1990, of cyclophosphamide (100 mg/day).
His final illness began in October 1990 with a swollen calf, confirmed on venogram, as a femoral-popliteal deep vein thrombosis. Five days later, during the transition from intravenous heparin (APTT $=60 \mathrm{~s}$ ) to warfarin ( $\mathrm{INR}=1$ ), and having remained normotensive, he complained of acute severe headache. Cerebellar haemorrhage was the presumptive diagnosis when, over several hours, he developed right sided cerebellar signs, dysarthria, ataxia, nausea and vomiting. A reducing conscious level and bilateral extensor plantar responses prompted immediate air ambulance transfer to neurosurgical care. On arrival, brain CT scan suggested a right cerebellar hemisphere infarct. However, he deteriorated rapidly and further CT scans demonstrated acute obstructive hydrocephalus changes. He died the following day, despite insertion of external ventricular drains.

\section{PATHOLOGY}

Marked pulmonary oedema and a right calf deep vein thrombosis were significant extracranial necropsy findings. The heart was normal, apart from evidence of old fibrinous pericarditis. There was no sign of systemic vasculitis.

The fresh brain (1580 gm) was swollen and surgical drains were present. The right cerebellar hemisphere contained an area of softening which extended into the right side of the pons. This region was the centre of striking histological abnormalities that extended into the midbrain and medulla. Collections of polymorph neutrophils and small haemorrhages in infarcted parenchyma surrounded many small blood vessels, which showed prominent vasculitic changes with fibrinoid necrosis (fig 2). Most of these vessels were small venules and several vascular territories were affected,

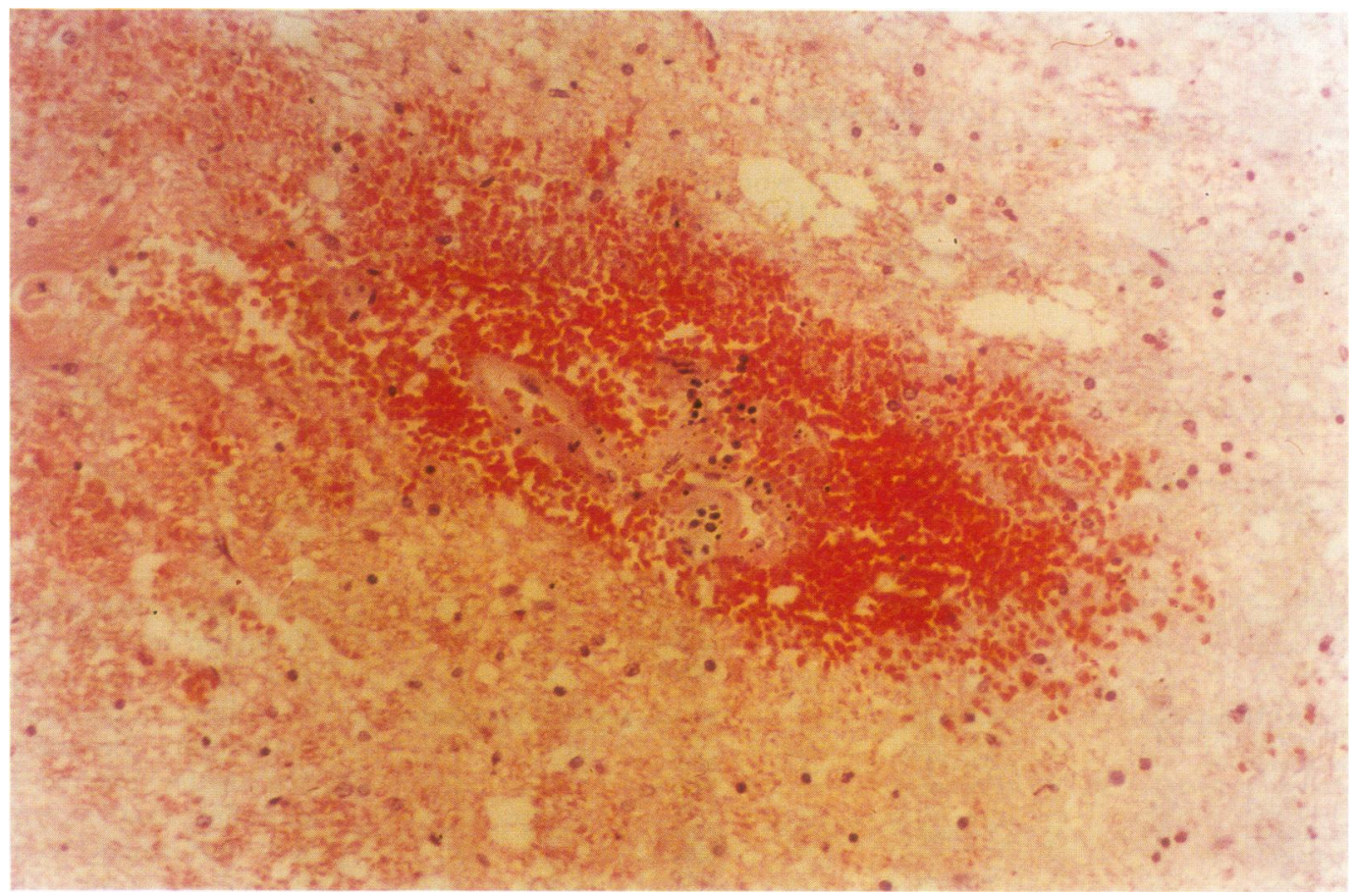

Figure 1 Perivascular haemorrhage in the midbrain (Haematoxylin and Eosin) 


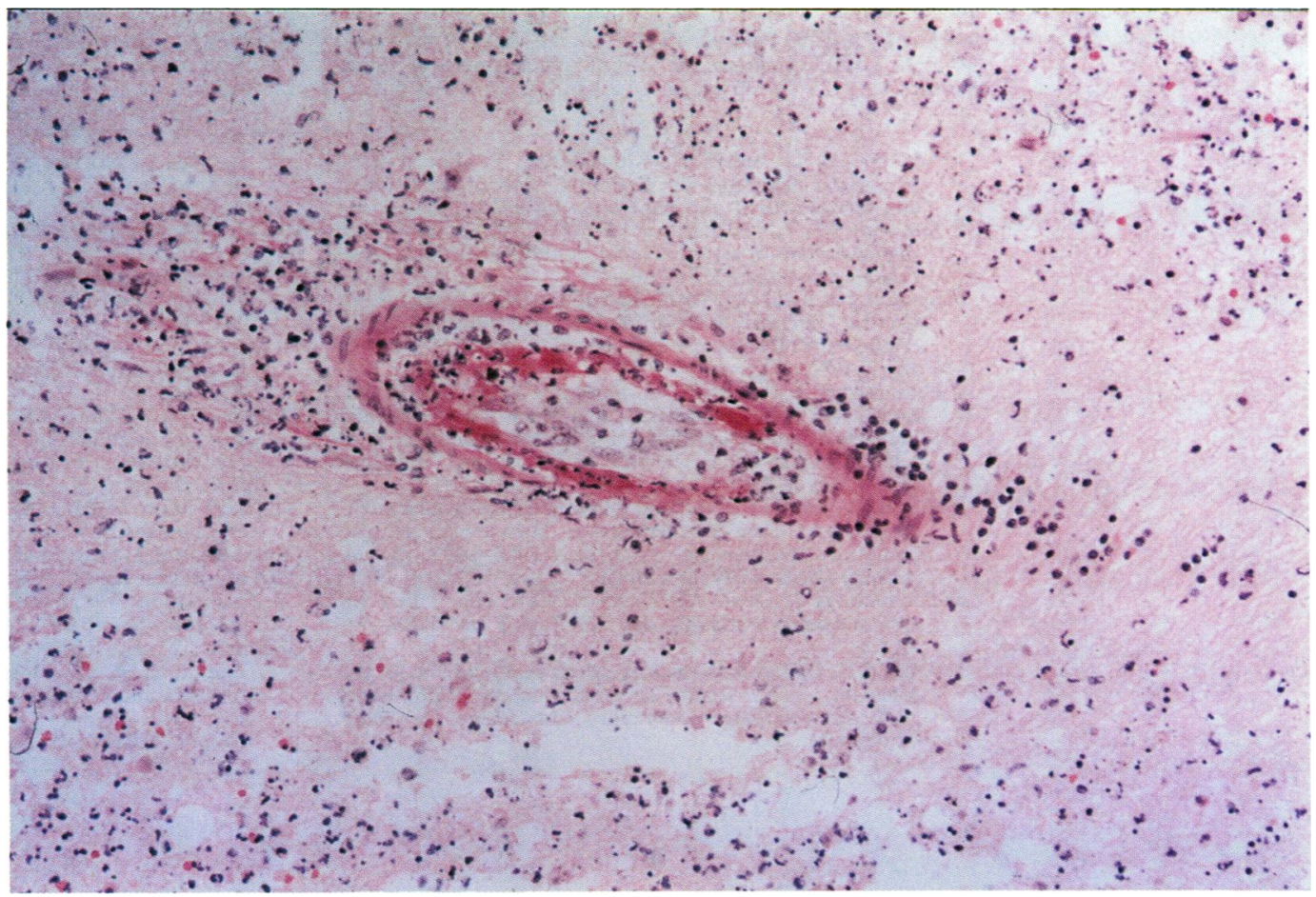

Figure 2 Vasculitis and fibrinoid necrosis of a cerebeller vessel with an inflammatory infiltrate in the surrounding brain (Haematoxylin and Eosin)

but no abnormality was found in the cerebral hemispheres.

\section{Discussion}

NP-SLE may result in various focal neurological signs. ${ }^{1}$ Cerebellar manifestations are distinctly uncommon, but well recognised. They may occur either in isolation or in association with brainstem and/or more widespread neurological involvement. ${ }^{2-8}$

The heterogeneous nature of NP-SLE suggests that different disease mechanisms may operate. Deposition of immune complexes in the choroid plexus, the effects of specific antibodies such as aCL, some perhaps resulting in direct neural cell dysfunction, and immune-mediated vascular disease have each been invoked as pathogenic. ${ }^{1}$ Large vessel vasculitis and embolic disease from a LibmanSachs endocarditis have also been recorded in NP-SLE. ${ }^{10}$ Ischaemic lesions, however, especially micro-infarcts, are a common finding, and microvascular abnormalities have been found in up to $67 \%$ of patients. ${ }^{251112}$ Small vessel vasculitis was demonstrated by Johnson et al in 3/24 cases of NP-SLE, accounting for $3 / 6$ deaths attributed to neurological involvement, ${ }^{2}$ and in $4 / 57$ cases in another series. ${ }^{5}$ An exudative capillary vasculopathy with perivascular haemorrhages may also occur, described in $2 / 57$ cases by Ellis et al, ${ }^{5}$ who noted that earlier studies often failed to distinguish between true arteritis and perivascular inflammation. Recent work suggests that in longstanding NP-SLE, intramural platelet deposition may contribute to small vessel vasculopathy following repeated episodes of immune-mediated damage. ${ }^{13}$

In the absence of specific neurological signs, reliable tests or imaging modalities, the diagnosis of NP-SLE depends upon a high index of suspicion and the exclusion of other conditions. General indices of disease activity in SLE are often unreliable in NP-SLE, although the presence of cutaneous or visceral vasculitis may identify patients at risk. ${ }^{4}$.The APS can occur in association with SLE, and may itself result in neurological manifestations. ${ }^{14}$ Livedo reticularis and splinter haemorrhages were evident in case 1 , and deep vein thromboses had occurred in both cases, all clinical features recognised in the APS. The aCL antibody had been identified and measured at an apparently constant and low level in case 1 since the onset of SLE, but this information was not available in case 2 .

Brain CT may demonstrate abnormalities in patients with NP-SLE, and while MRI is more sensitive, both may be normal. ${ }^{15}$ Neurophysiological tests may demonstrate neuronal dysfunction. Of the laboratory indices, a modest elevation of CSF protein is a common finding. ${ }^{1}$ Based predominantly on uncontrolled, retrospective and anecdotal data, the treatment of NP-SLE remains largely empirical. ${ }^{1}$ Case 1 deteriorated and died despite a combination of treatments, which included high dose immunosuppression. In case 2 , the diagnosis of NP-SLE was considered only at necropsy.

Detailed histopathological information from the brain in NP-SLE is rarely available. Both cases were unusual in that the clinical manifestations and distribution of pathological abnormalities were each confined to the cerebellum and brainstem, the reasons for which remain obscure. These cases demonstrate two distinct forms of small vessel vasculopathy seen in NP-SLE and suggest that this type of lesion may underlie severe forms of the disease. 
We thank Dr T W Parsons, Dr L Illis and Mr G Neil-Dwyer for their contributions to the clinical management of these patients.

1 Adelman D C, Saltiel E, Klinenberg J R. The neuropsychiatric manifestations of systemic lupus erythematosus: psychiatric manifestations of systemic lupus erythematosus:

2 Johnson R T, Richardson E P. The neurological manifestations of systemic lupus erythematosus. Medicine (Baltimore) 1968; 47

3 Sergent J S, Lockshin M D, Klempner M S, Lipsky B A. Central nervous system disease in systemic lupus erythematosus. Therapy and prognosis. $\mathrm{Am} \mathcal{F} \mathrm{Med} 1975$; 58: $644-54$.

4 Feinglass E J, Arnett F C, Dorsch C A, Zizic T M, Stevens M B. Neuropschiatric manifestations of systemic lupus erythematosus: diagnosis, clinical spectrum, and relationship to other features of the disease. Medicine (Baltimore) 1976; 55: 323-39.

5 Ellis S G, Verity M A. Central nervous system involvement in systemic lupus erythematosus: a review of neuroin systemic lupus erythematosus: a review of neuropathologic findings in 57 cas
Arthritis Rheum 1979; 8: $212-21$.

6 Tuchman A J, Daras M, David S. Cerebellar ataxia in systemic lupus erythematosus. NY State f Med 1983; 83: 983-4.

7 Singh R R, Prasad K, Kumar A, Misra A, Padmakumar K, Malaviya A N. Cerebellar ataxia in systemic lupus erythematosus: three case reports. Ann Rheum Dis 1988; 47: $954-6$.
8 Sibley J T, Olszynski W P, Decoteau W E, Sundaram M B. The incidence and prognosis of central nervous system
disease in systemic lupus erythematosus. $\mathcal{F}$ Rheumatol 1992; 19: 47-52.

9 Weiner D K, Allen N B. Large vessel vasculitis of the central nervous system in systemic lupus erythematosus: report and review of the literature. I Rheumatol 1991; 18: 748-51.

10 Devinsky O, Petito C K, Alonso D R. Clinical and neuropathological findings in systemic lupus erythematosus: the role of vasculitis, heart emboli and thrombotic thrombocytopenic purpura. Ann Neurol 1988; 23: $380-4$

1 Hanley J G, Walsh N M G, Sangalang V. Brain pathology in systemic lupus erythematosus. $\mathcal{F}$ Rheumatol 1992; 19: $732-41$.

12 Bunning R D, Laureno R, Barth W F. Florid central nervous system vasculitis in a fatal case of systemic lupus erythematosus. F Rheumatol 1982; 9: 735-8.

13 Ellison D, Gatter K, Heryet A, Esiri M. Intramural platelet deposition in cerebral vasculopathy of systemic lupus erythematosus. F Clin Path 1993; 46: 37-40.

14 Asherson R A, Derksen R H W M, Harris E N, et al. Chorea in systemic lupus erythematosus and 'lupus-like' disease: association with antiphospholipid antibodies. Semin association with antiphospholipid

15 Sibbitt W L, Sibbitt R R, Griffey R H, Eckel C, Bankhurst A D. Magnetic resonance and computed tomographic imaging in the evaluation of acute neuropsychiatric disease in systemic lupus erythematosus. Ann Rheum Dis 1989; 48: 1014-22. 